ARTIGO ESPECIAL / SPECIAL ARTICLE

\title{
Fatores associados a maior risco de ocorrência de óbito por COVID-19: análise de sobrevivência com base em casos confirmados
}

\author{
Factors associated with increased risk of death from COVID-19: a survival \\ analysis based on confirmed cases
}

Maria Helena Rodrigues Galvão' (D), Angelo Giuseppe Roncalli'

RESUMO: Objetivo: Realizar uma análise de sobrevivência de indivíduos diagnosticados com COVID-19 identificados pelos sistemas de informação em saúde, analisando os fatores associados ao maior risco de ocorrência de óbitos. Métodos: Análise de sobrevivência de indivíduos notificados por COVID-19 no estado do Rio Grande do Norte até o dia 24 de agosto de 2020, utilizando dados dos sistemas de informação em saúde. A variável dependente foi o tempo até a ocorrência do desfecho. As variáveis independentes foram sexo, cor da pele, faixa etária, residir ou não na capital e presença de comorbidades. Para análise de dados, utilizou-se o método de Kaplan-Meyer e, na análise multivariada, utilizou-se o modelo de regressão de Cox tempo-dependente, utilizando a covariável "tempo desde a notificação do evento em dias". Resultados: Apresentaram maiores riscos de ocorrência de óbitos por COVID-19 os indivíduos com 80 anos ou mais de idade $(\mathrm{HR}=8,06 ; \mathrm{p}<0,001)$, do sexo masculino ( $\mathrm{HR}=1,45 ; \mathrm{p}<0,001)$, com cor de pele não branca $(\mathrm{HR}=$ 1,$13 ; \mathrm{p}<0,033$ ) ou sem informação ( $\mathrm{HR}=1,29 ; \mathrm{p}<0,001$ ), que tinham comorbidades ( $\mathrm{HR}=10,44 ; \mathrm{p}<0,001$ ) ou que a presença de comorbidades não foi informada ( $\mathrm{HR}=10,87 ; \mathrm{p}<0,001)$. Conclusão: $\mathrm{O}$ maior risco de ocorrência de óbitos por COVID-19 foi observado em indivíduos idosos, sobretudo os com idade acima de 80 anos, pacientes com comorbidades, homens e com cor de pele não branca. Com base na identificação desse perfil, estratégias e linhas de cuidado específicas devem ser tomadas para prevenir a evolução ao óbito nesses casos pela identificação desses indivíduos no sistema de saúde.

Palavras-chave: Epidemiologia. Análise de sobrevida. Infecções por coronavírus.

'Programa de Pós-Graduação em Saúde Coletiva, Universidade Federal do Rio Grande do Norte - Natal (RN), Brasil.

Autor correspondente: Maria Helena Rodrigues Galvão. Programa de Pós-Graduação em Saúde Coletiva, Departamento de Odontologia, Universidade Federal do Rio Grande do Norte. Avenida Senador Salgado Filho, 1.787, Lagoa Nova, CEP: 59056-000, Natal, RN, Brasil. E-mail: mhrgalvao@gmail.com

Conflito de interesses: nada a declarar - Fonte de financiamento: Coordenação de Aperfeiçoamento de Pessoal de Nível Superior Brasil (CAPES) - Código de Financiamento 001. 
ABSTRACT: Objective: To perform a survival analysis of individuals diagnosed with COVID-19 identified by health information systems, analyzing the factors associated with the highest risk of death. Methods: Survival analysis of individuals notified with COVID-19 in Rio Grande do Norte State using data from the Health Information Systems for the surveillance of cases of and deaths from COVID-19. The dependent variable was the period until the outcome occurrence. The independent variables were sex, self-reported skin color, age group, residence in the capital, and the presence of comorbidities. For data analysis the Kaplan-Meyer method and Cox-time-dependent Regression Model for multivariate analysis were used, with the covariable "period since the event notification recorded in days". Results: Highest risk of death were observed in individuals aged 80 or older $(\mathrm{HR}=8.06$; $\mathrm{p}<$ $0.001)$, male $(\mathrm{HR}=1.45, \mathrm{p}<0.001)$, non-white skin color $(\mathrm{HR}=1.13 ; \mathrm{p}<0.033)$ or with no information $(\mathrm{HR}=$ 1.29; $\mathrm{p}<0.001$ ), with comorbidities (HR $=10.44 ; \mathrm{p}<0.001)$ or presence of comorbidities not reported $(\mathrm{HR}=$ 10.87; $\mathrm{p}<0.001)$. Conclusion: The highest risk of occurrence of deaths from COVID-19 was observed in older adults, especially those over 80 , patients who have comorbidities, men, and of non-white skin color. From the identification of the profile of patients with a higher risk of death with the identification by the health system, specific strategies of health care must be taken to prevent the evolution to death in these cases.

Keywords: Epidemiology. Survival analysis. Coronavirus infections.

\section{INTRODUÇÃo}

Em dezembro de 2019, a cidade de Wuhan, na China, reportou um surto de pneumonia de causa desconhecida que rapidamente se espalhou pelo país. O patógeno causador da doença foi identificado como um novo coronavírus, denominado como SARS-CoV-2, ou coronavírus da síndrome respiratória aguda grave-2. A Organização Mundial da Saúde (OMS) nomeou a doença causada pelo novo vírus de COVID-19 (coronavirus disease, ano de 2019), que foi declarada uma emergência de saúde pública de importância internacional, em 30 de janeiro de 2020, e, em 11 de março de 2020, como uma pandemia ${ }^{1}$. Desde então, iniciou-se um esforço global na produção de informações com a finalidade de desvendar os aspectos clínicos, epidemiológicos e fatores prognósticos da doença.

Em 22 de janeiro de 2020, a Secretaria de Vigilância em Saúde do Ministério da Saúde do Brasil divulgou as primeiras medidas de controle da infecção pelo até então novo coronavírus, com os sinais e sintomas, os critérios de definição de casos suspeitos e o fluxo de notificação, que inicialmente se deu mediante o Centro de Informações Estratégicas de Vigilância em Saúde Nacional, migrando para uma plataforma virtual e, posteriormente, pelo Sistema de Registro de Notificações do Ministério da Saúde (e-SUS Notifica), sendo recomendada a notificação imediata de casos suspeitos, prováveis e confirmados da COVID-192.

Após oito meses, segundo dados da OMS em 25 de agosto de 2020, já existiam mais de 23 milhões de casos confirmados e 810.492 óbitos decorrentes da doença no mundo. O Brasil é o segundo país com maior número de casos e óbitos pela doença no mundo, com mais de 3,5 milhões de casos confirmados e 115.309 óbitos $^{3}$. O estado do Rio Grande do Norte, 
cenário desta pesquisa, teve o seu primeiro caso confirmado em 12 de março de 2020, e acumula 60.161 casos confirmados e 2.192 óbitos $^{4}$.

Com relação aos fatores de risco para o agravamento da doença, uma revisão demonstrou que os casos mais graves da doença que evoluíram para pneumonia tiveram maior probabilidade de serem de pacientes mais velhos, do sexo masculino e com comorbidades, comparados aos casos mais leves. Contudo mais estudos foram recomendados para esclarecer as características epidemiológicas da COVID-19, bem como identificar os fatores de risco e o prognóstico dos pacientes infectados com o vírus SARS-CoV-2 ${ }^{1}$.

A maior parte dos estudos desenvolvidos até o presente momento foi proveniente de cenários internacionais e de contexto clínico-hospitalar ${ }^{5}$. Desse modo, ressalta-se a importância da produção de informações que demonstrem as características epidemiológicos da doença em contexto brasileiro, utilizando, para tal, os dados produzidos pelos sistemas de informação em saúde. Dessa forma, o presente artigo tem o objetivo de realizar uma análise de sobrevivência de indivíduos diagnosticados com COVID-19 identificados pelos sistemas de informação em saúde, analisando os fatores associados ao maior risco de ocorrência de óbitos.

\section{MÉTODOS}

Trata-se de estudo com dados secundários de análise de sobrevivência em indivíduos notificados por COVID-19 no estado do Rio Grande do Norte, Região Nordeste do Brasil. A fonte consistiu em dados consolidados dos sistemas de informação em saúde para a vigilância de casos e óbitos por COVID-19 em nível estadual. A Secretaria de Estado da Saúde Pública do Rio Grande do Norte (SESAP-RN) compõe sua base de dados da COVID-19 basicamente pela combinação de diferentes sistemas, notificados até 24 de agosto de 2020 (https: / / portalcovid19.saude.rn.gov.br/\#dados).:

- e-SUS Vigilância Epidemiológica (e-SUS-VE);

- Sistema de Informação de Vigilância da Gripe (SIVEP-Gripe);

- Sistema Gerenciador de Ambiente Laboratorial (GAL).

Realizou-se este estudo com informações disponíveis em domínio público, por meio de um conjunto de dados, não necessitando de aprovação ética.

A variável dependente consistiu no tempo de observação em dias até a ocorrência do desfecho (óbito por COVID-19) a partir da detecção desse paciente nos sistemas de informação para vigilância epidemiológica do agravo. O tempo de observação máximo foi de 165 dias e equivale ao tempo decorrido desde a identificação do primeiro caso de COVID-19 no estado.

Para a composição da variável dependente, considerou-se como a data de entrada no estudo a "data dos primeiros sintomas consolidada", que se trata da data de detecção dos primeiros sintomas de COVID-19 pelo sistema de saúde, e a "data de encerramento", que representa a data de ocorrência do óbito ou o fim do tempo de observação. O tempo decorrido até o desfecho foi medido em dias até o evento. 
Consideraram-se como critério de inclusão os casos confirmados notificados nos sistemas de informação até o dia 10 de agosto, tendo em conta um período de 14 dias anterior ao período final de observação. O critério foi adotado com o objetivo de eliminar os casos contidos no período de instabilidade dos dados para a investigação do desfecho óbito. Incluíram-se óbitos ocorridos até o dia 24 de agosto para os casos notificados até o período definido, considerando o tempo de observação estabelecido. Quanto ao critério para confirmação dos casos, incluíram-se apenas os casos de COVID-19 confirmados mediante teste diagnóstico do tipo RT-PCR, teste rápido para detecção de anticorpos IgM e IgG ou imunoensaio.

Atribuiu-se como critério de exclusão para a análise de sobrevivência os casos registrados mediante declaração de óbitos (death certificate only), em que a notificação do óbito ocorreu no dia ou após a detecção dos primeiros sintomas do indivíduo pelo sistema de saúde. A observação dos casos deu-se mediante seguimento passivo ${ }^{6}$.

Com a finalidade de identificar os fatores de risco e os fatores prognósticos para a ocorrência de óbitos por COVID-19, o tempo de sobrevivência foi modelado com base nas variáveis independentes:

- faixa etária (até 59 anos, de 60 a 79 anos ou 80 anos ou mais);

- cor da pele (branco, não branco ou sem informação);

- sexo (masculino, feminino);

- presença de comorbidades (sim, não ou sem informação);

- residir na capital ou em municípios do interior.

Para a análise de dados, empregou-se o método de Kaplan-Meyer, utilizando o teste log-rank, para a comparação de curvas de sobrevivência entre dois grupos, e o teste de Breslow, para a comparação de curvas de sobrevivência entre três grupos. Após a avaliação da proporcionalidade dos riscos por meio da avaliação dos resíduos de Schoenfeld, observou-se a ausência de proporcionalidade dos riscos no decorrer do período de observação e, consequentemente, a inadequação do modelo de regressão de Cox convencional para análise. Dessa forma, para a avaliação multivariada do efeito, utilizou-se o modelo de regressão de Cox tempo-dependente, utilizando, além da variável dependente, uma covariável tempo-dependente "tempo desde a notificação do evento em dias". Essa variável representa os diferentes momentos da epidemia no estado, tendo se mostrado estatisticamente significante para estimação da hazard ratio (HR) bruta e ajustada no modelo final, segundo os grupos de interesse. Estabeleceram-se como parâmetro para permanência no modelo final as variáveis que apresentaram significância estatística ao nível de 5\% pela estatística $z$ de Wald.

\section{RESULTADOS}

Durante o período de seguimento, foram notificados 52.607 casos e 1.842 óbitos por COVID19 no estado. A maior parte dos casos ocorreu em indivíduos de até 59 anos (81,0\%), de cor de pele não branca $(59,5 \%)$, sexo feminino $(52,8 \%)$, residentes em municípios do interior $(61,7 \%)$ e que não tinham comorbidades $(75,7 \%)$. Com relação aos óbitos, a maior parte ocorreu em 
indivíduos com faixa etária entre 60 e 79 anos (43,2\%), cor de pele não branca (50,9\%), sexo masculino (55,4\%), residentes no interior $(54,6 \%)$ e com comorbidades $(69,4 \%)$. Maior letalidade foi observada em idosos com 80 anos ou mais $(27,6 \%)$, em indivíduos com comorbidades $(12,4 \%)$ e sem informação sobre a presença de comorbidades (13,5\%) (Tabela 1).

Ao avaliar as curvas de sobrevivência durante o tempo de observação de 165 dias da epidemia, considerando como evento de interesse o óbito por COVID-19, a probabilidade de sobrevivência de indivíduos sem comorbidades ao fim do período de observação foi de $99,4 \%$, enquanto os indivíduos com comorbidades foi de $86,4 \%$. Ao considerar a faixa etária, a probabilidade de sobrevivência acumulada de indivíduos com até 59 anos foi de $98,7 \%$, enquanto, entre os indivíduos com 80 anos ou mais, foi de $69,7 \%$. Em indivíduos brancos e não brancos, a probabilidade de sobrevivência acumulada foi de $96,7 \%$, e, em indivíduos sem informação quanto à cor da pele, foi de $91,3 \%$. Os indivíduos do sexo feminino obtiveram probabilidade de sobrevivência de $96,8 \%$, superior aos do sexo masculino (95,5\%), ao fim do período de observação. Com relação ao município de residência, os indivíduos que residiam na capital apresentaram menor probabilidade de sobrevivência acumulada $(95,5 \%)$ que os indivíduos que residiam em municípios do interior (96,6\%). Observaram-se, para

Tabela 1. Frequências absolutas e relativas de casos, óbitos e letalidade por COVID-19 no estado do Rio Grande do Norte em 10 de agosto de 2020 (Brasil, 2020).

\begin{tabular}{|c|c|c|c|c|c|}
\hline & \multicolumn{2}{|c|}{ Casos de COVID-19 } & \multicolumn{2}{|c|}{ Óbitos por COVID-19 } & \multirow{2}{*}{$\begin{array}{c}\text { Letalidade } \\
\%\end{array}$} \\
\hline & $\mathrm{n}$ & $\%$ & $\mathrm{n}$ & $\%$ & \\
\hline Total & 52.607 & 100,0 & 1.842 & 100,0 & 3,5 \\
\hline \multicolumn{6}{|l|}{ Faixa etária } \\
\hline Até 59 anos & 42.634 & 81,0 & 520 & 28,2 & 1,2 \\
\hline 60 anos a 79 anos & 8.061 & 15,4 & 795 & 43,2 & 9,9 \\
\hline 80 anos ou mais & 1.912 & 3,6 & 527 & 28,6 & 27,6 \\
\hline \multicolumn{6}{|l|}{ Cor da pele } \\
\hline Branco & 15.746 & 29,9 & 446 & 24,2 & 2,8 \\
\hline Não branco & 31.271 & 59,5 & 938 & 50,9 & 3,0 \\
\hline Não informado & 5.590 & 10,6 & 458 & 24,9 & 8,2 \\
\hline \multicolumn{6}{|l|}{ Sexo } \\
\hline Feminino & 27.797 & 52,8 & 822 & 44,6 & 3,0 \\
\hline Masculino & 24.810 & 47,2 & 1.020 & 55,4 & 4,1 \\
\hline \multicolumn{6}{|l|}{ Município que reside } \\
\hline Capital & 20.147 & 38,3 & 837 & 45,4 & 4,2 \\
\hline Interior & 32.460 & 61,7 & 1.005 & 54,6 & 3,1 \\
\hline \multicolumn{6}{|c|}{ Presença de Comorbidades } \\
\hline Não & 39.830 & 75,7 & 234 & 12,7 & 0,6 \\
\hline Sim & 10.333 & 19,6 & 1.278 & 69,4 & 12,4 \\
\hline Não informado & 2.444 & 4,6 & 330 & 17,9 & 13,5 \\
\hline
\end{tabular}


todas as variáveis avaliadas, diferenças estatisticamente significantes entre as curvas de sobrevivência entre os grupos ( $\mathrm{p}<0,001)$ (Material Suplementar 1, 2 e 3 ).

$\mathrm{Na}$ análise não ajustada, observou-se que o efeito de todas as variáveis independentes foi significante para explicar o risco de ocorrência de óbitos por COVID-19. Após análise ajustada, as variáveis faixa etária, sexo, cor da pele e presença de comorbidades mantiveram-se significantes para explicar o risco de ocorrência de óbitos. Apresentaram maiores riscos de ocorrência de óbitos por COVID-19 os indivíduos com 80 anos ou mais de idade (HR = 8,06; $\mathrm{p}<$ 0,001), do sexo masculino ( $\mathrm{HR}=1,45 ; \mathrm{p}<0,001)$, com cor de pele não branca $(\mathrm{HR}=1,13 ; \mathrm{p}<$ $0,033$ ) ou sem informação (HR = 1,29; $\mathrm{p}<0,001)$, com comorbidades (HR = 10,44; $\mathrm{p}<0,001$ ) ou que a presença de comorbidades não foi informada $(\mathrm{HR}=10,87 ; \mathrm{p}<0,001)$ (Tabela 2).

Tabela 2. Análise de sobrevivência de casos de COVID-19 no estado do Rio Grande do Norte. Ajuste feito pela regressão múltipla de Cox com variável tempo dependente (Brasil, 2020).

\begin{tabular}{|c|c|c|c|c|}
\hline & \multicolumn{2}{|c|}{ Análise não ajustada } & \multicolumn{2}{|c|}{ Análise ajustada } \\
\hline & Valor $p$ & HR (IC95\%) & Valor $p$ & HR (IC95\%) \\
\hline \multicolumn{5}{|l|}{ Faixa etária } \\
\hline Até 59 anos & & 1 & & 1 \\
\hline 60 anos a 79 anos & $<0,001$ & $\begin{array}{c}7,43 \\
(6,65-8,31)\end{array}$ & $<0,001$ & $\begin{array}{c}3,87 \\
(3,45-4,34)\end{array}$ \\
\hline 80 anos ou mais & $<0,001$ & $\begin{array}{c}18,41 \\
(16,28-20,82)\end{array}$ & $<0,001$ & $\begin{array}{c}8,06 \\
(7,08-9,16)\end{array}$ \\
\hline \multicolumn{5}{|l|}{ Cor da pele } \\
\hline Branco & & 1 & & 1 \\
\hline Não branco & 0,046 & $\begin{array}{c}1,12 \\
(1,01-1,26)\end{array}$ & 0,033 & $\begin{array}{c}1,13 \\
(1,01-1,27)\end{array}$ \\
\hline Não informado & $<0,001$ & $\begin{array}{c}1,99 \\
(1,71-2,30)\end{array}$ & $<0,001$ & $\begin{array}{c}1,29 \\
(1,12-1,48)\end{array}$ \\
\hline \multicolumn{5}{|l|}{ Sexo } \\
\hline Feminino & & 1 & & 1 \\
\hline Masculino & $<0,001$ & $\begin{array}{c}1,49 \\
(1,35-1,63)\end{array}$ & $<0,001$ & $\begin{array}{c}1,45 \\
(1,32-1,59)\end{array}$ \\
\hline \multicolumn{5}{|l|}{ Município que reside } \\
\hline Capital & & 1 & - & - \\
\hline Interior & $<0,001$ & $\begin{array}{c}0,66 \\
(0,60-0,72)\end{array}$ & - & - \\
\hline \multicolumn{5}{|c|}{ Presença de Comorbidades } \\
\hline Não & & 1 & & 1 \\
\hline $\operatorname{Sim}$ & $<0,001$ & $\begin{array}{c}19,60 \\
(17,03-22,56)\end{array}$ & $<0,001$ & $\begin{array}{c}10,44 \\
(9,0112,09)\end{array}$ \\
\hline Não informado & $<0,001$ & $\begin{array}{c}14,30 \\
(12,61-17,68)\end{array}$ & $<0,001$ & $\begin{array}{c}10,87 \\
(9,16-12,90)\end{array}$ \\
\hline Covariável Tempo* & $<0,001$ & 1,00 & $<0,001$ & 1,00 \\
\hline
\end{tabular}

*Tempo de sobrevida (dias) × tempo desde a notificação (dias). Omnibus Test de significância do modelo: $\chi^{2}=12240,9$; $\mathrm{p}<$ 0,001; HR: hazard ratio; IC95\%: intervalo de confiança de $95 \%$. 


\section{DISCUSSÃo}

Os resultados do presente estudo revelaram quais os principais fatores que estão associados ao maior risco de ocorrência de óbito por COVID-19, entre os fatores avaliados, trazendo importantes subsídios para a tomada de decisão clínica e política, uma vez que permitem reconhecer os fatores associados ao prognóstico da doença no momento da identificação do caso pelo sistema de saúde.

Com relação à faixa etária, observou-se um efeito dose-resposta, em que os idosos entre 60 e 79 anos apesentaram risco 2,87 e idosos com 80 anos ou mais apresentaram risco 7,06 vezes maior de morrer por COVID-19 que indivíduos até 59 anos. Verificou-se a associação entre idade avançada e sintomas graves da COVID-19 em diversos estudos $\operatorname{clínicos}^{1,5,7}$. Ao comparar pacientes que desenvolveram síndrome respiratória aguda grave (SRAG) em decorrência da COVID-19 com pacientes sem SRAG, os pacientes que desenvolveram sintomas mais graves eram idosos, e a maioria destes tinha comorbidades. Além disso, notaram-se altas taxas de mortalidade nos pacientes com SRAG moderada ou severa ${ }^{7}$.

No Brasil, desde os primeiros meses de pandemia, tem-se observado que indivíduos idosos representam o maior percentual entre os óbitos por COVID-19, além disso, apresentam taxas de letalidade acumulada superiores às encontradas na população em geral. Também se verificou a influência de fatores sociodemográficos contextuais relacionados à raça e à renda nas taxas de mortalidade por COVID-19 na população idosa ${ }^{8}$.

A presença de comorbidades mostrou-se o fator com maior efeito para a ocorrência de óbitos por COVID-19. A presença de comorbidades aumenta o risco de óbito em 9,44 vezes em comparação aos indivíduos sem comorbidade. Embora a taxa de letalidade por COVID-19 seja mais baixa que a observada nas epidemias de doenças causadas por outros coronavírus, como síndrome respiratória aguda grave (SARS) e síndrome respiratória do Oriente Médio (MERS), observa-se uma letalidade aumentada em grupos específicos. Ao avaliar apenas os óbitos em decorrência da COVID-19, um estudo demonstrou que $31,5 \%$ dos casos tinham idade superior a 60 anos ou comorbidades 5 .

Dessa forma, a população idosa merece atenção especial por apresentar também maior vulnerabilidade para o desenvolvimento de comorbidades, como doenças cardiovasculares e cerebrovasculares, câncer e transtornos mentais, o que pode potencializar o risco de óbitos por COVID-19. Existe a necessidade de serem desenvolvidos protocolos preventivos específicos, tendo em vista a heterogeneidade da população idosa, com enfoque nos idosos com maior faixa etária e presença de comorbidades ${ }^{9}$.

Ser do sexo masculino foi um fator associado a um maior risco de morrer em decorrência da COVID-19. Homens apresentaram probabilidade de sobrevivência acumulada menor que mulheres, bem como um risco de óbito $45 \%$ maior que o das mulheres. Uma revisão sistemática, com metanálise incluindo nove estudos, demonstrou que os homens representam $60 \%$ dos pacientes com COVID-19, sugerindo maior susceptibilidade para 
infecção pelo vírus nessa população ${ }^{5}$. Contudo, no presente estudo, a maior proporção de casos observada foi do sexo feminino.

Esse achado, além de possuir relevância clínica com relação ao prognóstico da doença, também apresenta relevância para a política de saúde, tendo em vista que o sexo masculino historicamente tem menor acesso aos serviços de saúde. Além disso, a maior probabilidade de ocorrência de óbito em homens pode ser explicada por estes procurarem os serviços de saúde em casos mais graves ${ }^{10}$.

Apesar da importância clínica da variável presença de comorbidades para o prognóstico, observa-se que essa informação está ausente em 4,6\% dos casos notificados, tendo maior percentual de omissão em casos que evoluíram para óbito. A omissão dessa informação em casos de óbito fica evidente ao verificar a taxa de letalidade de $13,5 \%$ dos casos e o risco de ocorrência de óbito 9,87 vezes maior nesse grupo, em comparação aos casos sem comorbidades.

A presença de dados não informados também foi observada para a variável cor da pele, com 10,6\% dos casos não apresentando informações para essa variável. Isso evidencia o preenchimento inadequado do campo raça/cor nos sistemas de informação em saúde, o que prejudica a avaliação de condições de saúde com recorte étnico-racial $^{11}$. Apesar do percentual de ausência de informações nessa variável, optou-se por manter os dados sem informação com o objetivo de expressar a realidade dos sistemas de informação em saúde para vigilância de agravos.

A despeito da presença de casos sem informação de cor da pele, ter cor de pele não branca aumentou o risco de ocorrência de óbito por COVID-19 em 13\%, comparado aos indivíduos de pele branca. O efeito da etnia / cor da pele no risco de óbito por COVID-19 também foi notado em uma análise de sobrevivência de casos confirmados de COVID19 realizada no Reino Unido. Mesmo após o ajuste pelas variáveis sexo, idade, pobreza e região, indivíduos de etnias com cor de pele não branca tiveram risco de óbito até duas vezes maior que indivíduos brancos. Contudo o efeito da presença de comorbidades não foi incluído, o que pode ter interferido nos achados do estudo ${ }^{12}$.

Outro estudo conduzido no Reino Unido, com dados de pacientes com COVID-19 admitidos em hospital, observou maior admissão de indivíduos negros e de grupos étnicos minoritários comparados aos indivíduos brancos, com relação à proporção desses grupos na população. Ao avaliar a sobrevivência desses pacientes após 30 dias, os indivíduos negros e os grupos étnicos minoritários tiveram a mesma ou maior probabilidade de sobrevivência que indivíduos brancos. Além disso, não se constatou associação entre etnia e sobrevivência em pacientes hospitalizados por COVID-19, após ajustes por outros fatores incluindo a presença de comorbidades ${ }^{13}$.

No presente estudo, observou-se a manutenção do efeito da cor da pele para a predição do risco de óbito por COVID-19 após o ajuste das demais variáveis, incluindo a variável presença de comorbidades. Dessa forma, sugere-se que as pesquisas sobre mortalidade por COVID-19 devam considerar fatores biológicos e sociais, com a finalidade de identificar disparidades étnicas e raciais relacionadas ao agravo ${ }^{14}$. 
No intercurso da pandemia, várias intervenções em saúde pública foram necessárias. Entre estas medidas, adotaram-se restrições de deslocamento e lockdown em várias localidades, com a finalidade de achatar a curva da epidemia. O subsídio essencial para a tomada de decisões em saúde pública foram os dados produzidos, permitindo o surgimento da chamada "saúde pública de precisão"15.

Ante tal cenário, ficou mais evidente a importância dos sistemas de informação em saúde para a vigilância epidemiológica, com a finalidade de disponibilizar informações atualizadas sobre mortalidade e morbidade pela nova doença de maneira ágil e confiável para subsidiar ações de prevenção e controle de disseminação da nova doença, bem como a estruturação da rede de atenção à saúde para receber novos casos.

Um importante aspecto da qualidade dos sistemas de informação em saúde é o preenchimento adequado de cada variável no sistema. Contudo a completude dos dados nem sempre é observada, havendo uma lógica de relevância no preenchimento das informações, pelo pressuposto da importância atribuída a essa informação para o setor saúde. Dessa forma, algumas informações relevantes não são coletadas ou são dadas como “ignoradas". Nesse processo, algumas variáveis como sexo e idade são priorizadas e outras como raça/cor e escolaridade são ignoradas ${ }^{16}$. Esse fenômeno sofre influência especialmente da concepção de saúde adotada, que, em muitas situações, está desvinculada do conceito dos determinantes sociais da saúde e mais voltado para o modelo biomédico ${ }^{17}$.

O presente estudo apresenta como potencialidade a capacidade de produção de dados de sobrevivência por COVID-19 utilizando dados locais. As limitações do estudo estão relacionadas à utilização de dados secundários, que são passíveis de sofrer efeito do viés de informação, embora essa seja a fonte de dados mais comum para estudos com análise de sobrevivência ${ }^{6,13}$. Uma das estratégias metodológicas utilizadas para redução do viés foi a exclusão dos casos notificados nas duas últimas semanas do período de observação, que é considerado o período necessário para estabilidade dos casos. Além disso, realizou-se a inclusão do tempo desde a notificação com base no modelo de regressão tempo-dependente, com a finalidade controlar o viés tempo-concorrente durante os vários estágios da epidemia.

Observou-se o maior risco de ocorrência de óbitos por COVID-19 em indivíduos idosos, sobretudo os com idade acima de 80 anos, pacientes com comorbidades, homens e com cor de pele não branca. Após o ajuste pelas demais variáveis, não se verificou o efeito de residir em municípios do interior do estado no risco de óbito por COVID-19 no presente estudo. Atualmente, o efetivo controle de infecção é a única forma de prevenir a disseminação da SARS-CoV- $2^{1}$. Contudo, ao conhecer as populações com maior risco de agravamento e óbito pela doença, pode-se lançar mão de estratégias terapêuticas que previnam o agravamento da doença nessas populações. Além disso, contribui para que se prepare a rede de atenção hospitalar, a partir do momento do reconhecimento do perfil epidemiológico dos casos diagnosticados, prevendo a demanda de utilização de leitos hospitalares e óbitos em decorrência da doença. 


\section{REFERÊNCIAS}

1. Lai CC, Liu YH, Wang CY, Wang Y-H, Hsueh S-C, Yen $\mathrm{M}-\mathrm{Y}$, et al. Asymptomatic carrier state, acute respiratory disease, and pneumonia due to severe acute respiratory syndrome coronavirus 2 (SARS-CoV-2): Facts and myths. J Microbiol Immunol Infect 2020; 53(3): 404-12. http: / / doi.org/10.1016/j.jmii.2020.02.012

2. Brasil. Ministério da Saúde. Secretaria de Vigilância em Saúde. Boletim Epidemiológico $\mathrm{n}^{\circ} 4$ de janeiro de 2020 [Internet]. Brasil: Ministério da Saúde; 2020 [acessado em 14 ago. 2020]. Disponível em: https: / www.saude.gov.br/ images/pdf/2020/janeiro/23/Boletim_epidemiologico_ SVS_04.pdf

3. World Health Organization. Coronavirus disease (covid19) [Internet]. Genebra: World Health Organization; 2020 [acessado em 26 ago. 2020]. Disponível em: https: / / www. who.int/emergencies/diseases/novel-coronavirus-2019

4. Rio Grande do Norte. Secretaria de Estado da Saúde Pública. Informações importantes para a população em geral e profissionais de saúde sobre o novo coronavírus [Internet]. Rio Grande do Norte: Secretaria de Estado da Saúde Pública [acessado em 26 ago. 2020]. Disponível em: https://portalcovid19.saude.rn.gov.br/

5. LiL-Q, Huang T, Wang Y-Q, WangZ-P, Liang Y,Huang T-B, et al. Covid-19 patients' clinical characteristics, discharge rate, and fatality rate of meta-analysis. J Med Virol 2020; 92(6): 577-83. http:// doi.org/10.1002/jmv.25757

6. Bustamante-Teixeira MT, Faerstein E, Latorre MR. Técnicas de análise de sobrevida. Cad Saude Publica 2002; 18(3): 57994. http:/ / doi.org/10.1590/S0102-311X2002000300003

7. Liu Y, Sun W, Li J, Chen L, Wang Y, Zhang L, et al. Clinical features and progression of acute respiratory distress syndrome in coronavirus disease 2019 . medRxiv 2020: 2020.02.17.20024166. http:// doi. org/10.1101/2020.02.17.20024166

8. Barbosa IR, Galvão MHR, Souza TA, Gomes SM, Medeiros A de A, Lima KC. Incidência e mortalidade por covid-19 na população idosa brasileira e sua relação com indicadores contextuais: um estudo ecológico. Rev Bras Geriatr Gerontol 2020; 23(1): 200171. http:/ / doi. org/10.1590/1981-22562020023.200171

9. Zaslavsky C, Gus I. Idoso. Doença cardíaca e comorbidades. Arq Bras Cardiol 2002; 79(6): 635-9. http:// doi. org/10.1590/s0066-782x2002001500011

10. Teixeira DBS. Atenção à saúde do homem: análise da sua resistência na procura dos serviços de saúde. Rev Cubana Enferm [Internet] 2016 [acessado em 18 ago. 2020]; 32(4). Disponível em: http:// revenfermeria.sld. $\mathrm{cu} /$ index.php/enf/article/view/985

11. Braz RM, Oliveira P de TR de, Reis AT dos, Machado NM da S. Avaliação da completude da variável raça/ cor nos sistemas nacionais de informação em saúde para aferição da equidade étnico-racial em indicadores usados pelo Índice de Desempenho do Sistema Único de Saúde. Saúde Debate 2013; 37(99): 554-62. http:/ / doi. org/10.1590/s0103-11042013000400002

12. Public Health England. Disparities in the Risk and Outcomes of covid-19 [Internet]. Londres: Public Health England; 2020 [acessado em 18 ago. 2020]. Disponível em: https: / assets.publishing.service.gov.uk/government/ uploads/system/uploads/attachment_data/file/908434/ Disparities_in_the_risk_and_outcomes_of_COVID_ August_2020_update.pdf

13. Harrison E, Docherty A, Semple C. Investigating Associations between Ethnicity and Outcome from covid-19 [Internet]. 2020 [acessado em 18 ago. 2020]. Disponível em: https: / / assets.publishing.service.gov.uk/ government/uploads/system/uploads/attachment_data/ file / 886433/s0238-co-cin-report-ethnicity-outcomes250420-sage29.pdf

14. Ravi K. Ethnic disparities in covid-19 mortality: are comorbidities to blame? Lancet 2020; 396(10243): 22. http: / / doi.org/10.1016/S0140-6736(20)31423-9

15. Rasmussen SA, Khoury MJ, del Rio C. Precision Public Health as a Key Tool in the covid-19 Response. JAMA 2020; 324(10): 933-4. http: / / doi.org/10.1001/ jama.2020.14992

16. Jorge MHPM, Laurenti R, Gotlieb SLD. Avaliação dos sistemas de informação em saúde no Brasil. Cad Saúde Colet [Internet] 2010 [acessdao em 18 ago. 2020]; 18(1): 7-18. Disponível em: http://www.cadernos.iesc.ufrj. br/cadernos/images/csc/2010_1/artigos/Modelo\%20 Livro\%20UFRJ\%201-a.pdf

17. Moraes IHS. Sistemas de Informação em Saúde:patrimônio da sociedade brasileira. In: Paim J, Almeida-Filho N, eds. Saúde Coletiva: Teoria e Prática. Rio de Janeiro: Medbook; 2014. p. 649-65.

\section{Recebido em: 09/09/2020 \\ Revisado em: 08/10/2020 \\ Aceito em: 29/10/2020 \\ Versão preprint em: 03/09/2020 \\ (https://doi.org/10.1590/SciELOPreprints.1175)}

Contribuição dos autores: MHRG contribuiu para a concepção do estudo, análise e interpretação dos dados do trabalho, elaboração das versões preliminares do artigo e aprovação final da versão a ser publicada. AGR contribuiu para a concepção do estudo, análise e interpretação dos dados do trabalho, revisão crítica do artigo e aprovação final da versão a ser publicada. 